\title{
Modeling and analysis of collective management of water resources
}

\author{
A. Tilmant ${ }^{1}$, P. van der Zaag ${ }^{1,2}$, and P. Fortemps ${ }^{3}$ \\ ${ }^{1}$ UNESCO-IHE, Department of Management and Institution, Delft, The Netherlands \\ ${ }^{2}$ Water Resources Section, Delft University of Technology, Delft, The Netherlands \\ ${ }^{3}$ Faculté Polytechnique de Mons, Department of Applied Mathematics and Operational Research, Mons, Belgium
}

Received: 4 July 2006 - Published in Hydrol. Earth Syst. Sci. Discuss.: 4 September 2006

Revised: 7 December 2006 - Accepted: 11 December 2006 - Published: 17 January 2007

\begin{abstract}
Integrated Water Resources Management (IWRM) recommends, among other things, that the management of water resources systems be carried out at the lowest appropriate level in order to increase the transparency, acceptability and efficiency of the decision-making process. Empowering water users and stakeholders transforms the decision-making process by enlarging the number of point of views that must be considered as well as the set of rules through which decisions are taken. This paper investigates the impact of different group decision-making approaches on the operating policies of a water resource. To achieve this, the water resource allocation problem is formulated as an optimization problem which seeks to maximize the aggregated satisfaction of various water users corresponding to different approaches to collective choice, namely the utilitarian and the egalitarian ones. The optimal operating policies are then used in simulation and compared. The concepts are illustrated with a multipurpose reservoir in Chile. The analysis of simulation results reveals that if this reservoir were to be managed by its water users, both approaches to collective choice would yield significantly different operating policies. The paper concludes that the transfer of management to water users must be carefully implemented if a reasonable trade-off between equity and efficiency is to be achieved.
\end{abstract}

\section{Introduction}

The management of large-scale water resources, such as multipurpose reservoirs, has traditionally been carried out by public administration. The motivations for a public management are both political and financial: to promote equity and arbitrate conflicts among water users, to maintain ownership on a strategic infrastructure, to ensure that certain water ser-

Correspondence to: A. Tilmant

(a.tilmant@unesco-ihe.org) vices viewed as public goods are preserved, and to promote the development of water resources which are highly capital intensive with long time investments (Dinar et al., 1997). As pointed out recently by various authors (Hjorth et al., 1998; WCD, 2000) the performance of many multipurpose schemes has not met the expected targets. Consequently, a (partial) transfer of management to water users is often cited as a potential alternative to a public (centrally-organized) administration.

This paper investigates the impacts of different approaches to collective choice on the management of a water resource system. More specifically, an optimization model is developed to determines optimal release policies for a multipurpose reservoir while explicitly considering multiple water users and different group decision-making approaches. The model seeks to maximize the aggregated satisfaction of the different water users and relies on flexible constraints to quantitatively model preferences formulated by the water users. The use of flexible constraints instead of objective functions is motivated by the fact that a flexible constraint includes a threshold below which a solution will be rejected and a threshold above which solutions are equally feasible (Dubois et al., 1996). In other words, between these two thresholds, the flexible constraint behaves as a local objective. In addition, flexible constraints also remove barrier language for non-technical water users since they can be described by linguistic variables and mathematically encoded as fuzzy sets. The idea here is that fuzzy sets are used as a vehicle to capture and process linguistically described water users' preferences formulated by both technical and nontechnical water users. In this paper, the terms "stakeholder", "water user", "actor" and "objective" play the same role: supplying preferences across alternatives. They can therefore be used interchangeably.

The optimal decision is defined as the solution for which the global satisfaction of the different water users is maximal. An aggregation of water users' preferences is therefore

Published by Copernicus GmbH on behalf of the European Geosciences Union. 
needed and must be carried out taking into account the complex rules that characterize the group decision-making. This paper illustrates the impact of these decision rules on the performance of a multipurpose reservoir as if it were managed by the water users. The second section is devoted to flexible constraints and objectives. Section 3 presents various flexible constraints satisfaction problems and how they can be interpreted in social choice theory. Then, the optimization of reservoir operation is discussed in Sect. 4 and the case study introduced in Sect. 5. Simulation results associated to various group decision-making approaches are analyzed in Sect. 6. Finally, concluding remarks are presented.

\section{Flexible constraints versus objectives}

Many water resources systems have been developed and managed to meet the demands for water formulated by multiple users, some of them with multiple objectives. Unfortunately, these objectives can seldom be fully satisfied due to the presence of various constraints, which can be of physical, legal or financial nature. The major difference between constraints and objectives comes from the imperativeness with which they must be satisfied: satisfying a constraint is more imperative than satisfying an objective (Slany, 1996). An objective function, on the other hand, associates to each alternative (or solution) $r$ a value $g(r)$ so as to provide a complete ranking of the set of feasible solutions: $r \succ_{g} r^{\prime}$ if and only if $g(r) \geq g\left(r^{\prime}\right)$, which means "the objective $g$ prefers $r$ to $r^{\prime \prime}$ ".

The notion of flexible (fuzzy) constraint is an attempt to represent both constraints and objectives by fuzzy sets. Let $\Re$ be the set of all solutions (feasible, non-feasible or partially feasible) and $O$ a fuzzy relation related to constraint $C$. A flexible constraint $C$ can be described by a fuzzy set defined by $O$ in $\Re$. For a solution $r \in \mathfrak{R}$, the membership grade $\mu_{O}(r)$ associated by $O$ to $r$ specifies the satisfaction level of $r$ according to the constraint $C$ :

$\mu_{O}(r)=1$. means $r$ totally satisfies $C$

$\left.\mu_{O}(r) \in\right] 0,1[$. means $r$ partially satisfies $C$

$\mu_{O}(r)=0$. means $r$ totally violates $C$

$\mu_{O}(r) \geq \mu_{O}\left(r^{\prime}\right)$. means $r$ is preferred to $r^{\prime}$

For a classical constraint, the relation $O$ is binary, whereas for a flexible constraint, $O$ is a fuzzy relation. In other words, $O$ is the fuzzy set of solutions more or less satisfying the flexible constraint $C$. The relation $O$ therefore rank-orders the feasible solutions like an objective function. But the flexible constraint differs from the objective function in that it also models a threshold below which a solution will be rejected and a threshold above which solutions are equally feasible. In a sense, a flexible constraint can be viewed as the association of a constraint and a criterion; the former defines the support of the flexible constraint, while the latter rank-orders the solutions according to preferences (Dubois et al., 1996).
The methodology described in this paper heavily relies on preferences formulated by water users. These preferences will be encoded as fuzzy sets in the optimization algorithm. More specifically, the membership grade $\mu$ translates water user satisfaction on the unit interval: the membership grade is 1 when the alternative completely satisfies the water user, and it is equal to 0 when the solution is completely rejected by the same water user. In the ]0, 1[ interval, solutions are partially accepted/rejected. Note that the term satisfaction has a logical meaning here: to "satisfy" a preference means that the prior conditions defining the preference are met. This definition has therefore no psychological sense in that it does not reflect a mental state of pleasure.

\section{Flexible constraint satisfaction problems}

Classical Constraint Satisfaction Problems (CSP) rely on "crisp" (hard) constraints: constraints can either be satisfied or not. In CSP, a solution must satisfy all constraints of the problem. A problem is said to be "underconstrained" if it accepts more than one solution. In that case, the decision maker has to consider other constraints to differentiate between these solutions. In contrast, when there is no solution, the problem is said to be "overconstrained" and one or more constraints must be relaxed. The Flexible Constraint Satisfaction Problem (FCSP) generalizes CSP by substituting at least one hard constraint by a flexible constraint. In a sense, this allows the decision maker to introduce an objective function in the problem, which can be used to relax an "overconstrained" problem, or to differentiate the solutions of an "underconstrained" problem.

Define $\mathfrak{C}$ as a finite set of flexible constraints $\left\{C_{1}, C_{2}, \ldots, C_{J}\right\}, \quad \mathfrak{O}$ as a finite set of fuzzy relations $\left\{O_{1}, O_{2}, \ldots, O_{J}\right\}$ and $r$ as an alternative $(r \in \Re)$. Each alternative $r$ is associated with a profile $\mu_{r}=\left(\mu_{O_{1}}(r), \mu_{O_{2}}(r), \ldots, \mu_{O_{J}}(r)\right) \in[0,1]^{J}$, where $\mu_{O_{j}}(r)$ is the satisfaction level of the $j$-th flexible constraint associated with the alternative $r$.

To rank the alternatives or select an alternative that best satisfies the flexible constraints, one can compute the global scores $D(r)$ from the profile of any alternative $r$ using an aggregated satisfaction $D: \mathbb{R}^{J} \rightarrow \mathbb{R}$. In classical constraint satisfaction problem, the most common definition of the aggregated satisfaction $D$ is based on the minimum operator (the min-ordering):

$D(r)=\mu_{O_{1} \cap O_{2} \cap \ldots \cap O_{J}}(r)=\min _{C_{j} \in \mathfrak{C}} \mu_{O_{j}}(r)$

The minimum operator belongs to a family of operators called triangular norms which have interesting mathematical properties (Zimmermann, 1991).

The best solutions $r^{*}$ are therefore those for which the aggregated satisfaction $D$ is maximum:

$r^{*}=\max _{r \in \Re} \min _{C_{j} \in \mathfrak{C}^{\mathfrak{C}}} \mu_{O_{j}}(r)$ 
The solutions $r^{*}$ maximize the satisfaction level of the least satisfied constraint, and therefore the FCSP is a max-min optimization problem (max-min solutions). The use of the min operator has been advocated by (Bellman and Zadeh, 1970). They adopted a logic point of view, interpreting the intersection of two fuzzy sets (which is obtained by combining with the min operator their membership grade functions) as the "logical AND" between two statements. It must be pointed out that this max-min approach is coherent with the properties of a flexible constraint, namely it cannot be violated. Any solution with a zero satisfaction level for a single constraint is rejected as impossible.

In social choice theory, the min-ordering corresponds to an egalitarian approach: every member (water user) has a veto power like the permanent members of the UN Security council. As soon as one user rejects a solution, it is globally rejected by the whole group.

Despite its pleasing mathematical properties, the minordering has two major drawbacks:

- it lacks of discrimination power by concentrating on the worst aspects of the alternatives;

- maxmin solutions are not Pareto optimal, meaning that the satisfaction of other water users could still be improved without deteriorating the satisfaction of the least-satisfied water user.

The first issue has been addressed by several authors, (e.g. Dubois et al., 1996; Dubois and Fortemps, 1999), who have proposed various refinements such as the leximin or the discrimin orderings. The second drawback has a practical dimension; the fact that Pareto optimality cannot be guaranteed implies that certain water users will not easily accept the solutions since the resource is not efficiently managed. However, as the solutions are taken on the basis of the leastsatisfied water user, they are consistent with the notion of justice as discussed by Rawls (1970).

An alternative definition of the aggregated satisfaction $D$ is based on the maximum operator. It represents a group decision-making in which each participant is a potential dictator (max-ordering). Here, as soon as a water user is fully satisfied with a solution, it is accepted by the whole group. In other words, this approach looks for the full satisfaction of at least one water user, nothwithstanding the satisfaction of the other users. Then the optimal solutions are the solutions of a maximax optimization problems (max-max solutions). However, the max-ordering suffers from the same drawbacks as the min-ordering.

In between these two extremes the utilitarian decisionmaking approach builds an additive satisfaction $D$ using, for example, a sum over all preferences. The solutions with the highest utility are the ones preferred (max-sum solutions):

$r^{*}=\max _{r \in \Re} \sum_{j} \mu_{O_{j}}(r)$
In contrast to max-min or max-max solutions, max-sum solutions are consistent with the tradition of multicriteria decision making and the idea of trade-off.

The utilitarian approach to collective choice also often implies that the actors, and thus their constraints, do no have the same importance. For example, diverting water for municipal water supply may be more important than for irrigation purposes. If a constraint is more important, it should play a more significant role in the aggregation function (Eq. 3).

Various methods exist for including relative importance. Here, the constraints are ordered with respect to each other by giving them a priority degree, which can be considered as a weight. In other words, the weight reflects the relative importance of the constraint as explained in Choo et al. (1999). We used Saaty's AHP method to generate a first set of weights, which was then adjusted by the experts familiar with the system. This procedure was made possible here because (i) the number of experts was limited and (ii) there was little room for interpretation of the relative importances as priorities are framed in the Chilean Water Code. In most water resources planning and management problems, however, the number of decision-makers is likely to be larger resulting in an heterogenous group in which a consensus on a weight vector will not easily emerge. In that case, one option might be to construct a separate hierarchy of the decision-makers but this issue is still under discussion in the literature (Srdjevic, 2007; Ramanathan, 2001; Honert and Lootsma, 1996).

Saaty's AHP method relies on pairwise comparisons between constraints to determine the weights. This procedure starts with the construction of a $\mathbf{J} \times \mathbf{J}$ matrix $\mathbf{A}$ in which the element $a_{i j}$ gives the relative importance of the constraint $C_{i}$ with respect to the constraint $C_{j}$. Note that the element $a_{j i}$ is calculated from $a_{j i}=1 / a_{i j}$ using Saaty arithmetic scale 19. Other scales could have been used such as the geometric scale (Lootsma, 1996), which uses the same semantic description as Saaty but with a different range $\left(e^{0 \gamma}\right.$ to $\left.e^{8 \gamma}\right)$ where $\gamma$ is a constant. The weight vector $\omega$ is then obtained from

$A \omega=\lambda_{\max } \omega$

where $\lambda_{\max }$ is the maximum eigenvalue of $A$. This vector is the importance associated with each of the constraints $C_{j}$ for $j \in[1,2, \ldots, J]$. More details about this methodology can be found in Saaty (1980). An alternative to the eigenvector technique is the Logarithmic Least Squares Technique (LLST), which chooses the weights that minimize the logarithmic square deviations (Beroggi, 1999).

We further assume that the weight factors are normalized according to

$\sum_{j} \omega_{j}=1, \quad \omega_{j} \in[0,1]$.

Once the weight vector is calculated, it can be introduced in the definition of the utilitarian, aggregated, satisfaction $D$ by, for example, directly multiplying the satisfaction level $\mu_{O_{j}}$ 
by its weight $\omega_{j}$. The optimization problem (Eq. 3) becomes the well-known weighted arithmetic mean:

$D^{p}(r)=\sum_{j} \omega_{j} \mu_{O_{j}}(r)$

Another option to aggregate the individual satisfaction levels $\mu_{O_{j}}$ is to use the weighted geometric mean (WGM). In fact, the multiplicative variant of AHP (Lootsma, 1996), which relies on WGM and LLST, could have been used as the WGM is more consistent with AHP in terms of priorities and judgements (Forman and Peniwati, 1998). However, we choose to work with the standard AHP because (i) the weighted arithmetic mean was felt to be more intuitive to the experts and (ii) the weight vector was "manually" adjusted by the experts so as to better reflect their perceptions. In practice, however, this last step is not mandatory and a sensitivity analysis with different weight vectors may be carried out.

The weighted arithmetic mean is, in turn, a particular case of the generalized averaging operator when the parameter $p=1$ (Kaymak and van Nauta Lemke, 1998)

$D^{p}(r)=\left\{\sum_{j} \omega_{j} \mu_{O_{j}}^{p}(r)\right\}^{1 / p} \quad, \quad p \in \mathbb{R} \backslash\{0\}$

and

$D^{0}(r)=\prod_{j} \mu_{O_{j}}(r)^{\omega_{j}}$

As a matter of fact, when $p \rightarrow \infty$ the aggregation function (Eq. 7) becomes equivalent to the max-operator, whereas when $p \rightarrow-\infty$ the aggregation is equivalent to the minopeator. In between these two extremes, the aggregation Function (7) can be tailored to model utilitarian decisionmaking. With the aggregation function (7), the best solution $r^{p, *}$ of the FCSP is given by

$r^{p, *}=\max _{r \in \Re}\left\{\sum_{j} \omega_{j} \mu_{O_{j}}^{p}(r)\right\}^{1 / p}, \quad p \in \mathbb{R} \backslash\{0\}$

and

$r^{0, *}=\max _{r \in \Re} \prod_{j} \mu_{O_{j}}(r)^{\omega_{j}}$

As mentioned early, the aggregation should also produce Pareto-optimal solutions. Utilitarian solutions (i.e. solutions corresponding to finite values of $p$ in Eq. 9) are all Pareto optimal, but notwithstanding this fact, these solutions might not necessarily be well perceived by water users since compensation between water users' satisfaction is assumed. In other words, a consensus might be difficult to reach, because certain water users might consider the resulting operating policy as a "zero-sum" game, while others might see it as a "positive-sum" game. It must be stressed here that the utilitarian and egalitarian conceptions of collective choice assume that:

- decision makers will choose among alternatives by articulating prior preferences;
- disagreement is the result of competition for the use of water.

The first assumption is often criticized by political scientists, because it fails to adequately take into account those water services that can be viewed as public goods (Sagoff, 1998): a typical example is flood control. In other words, these two conceptions only deal with "consumer" (water user) preferences and not "citizen" preferences.

\section{Reservoir operation optimization}

The different group decision-making approaches discussed in the previous section and their impacts on the management of a water resources are illustrated with a multipurpose reservoir operation problem. The reservoir operation problem typically consists in determining release decisions that should be made in order to meet the demands formulated by various water users/uses such as hydropower generation, flood control, low flow maintenance, recreation, irrigation, etc. Various models have been developed in the past to address this operation problem. The most common mathematical programming techniques are linear programming (LP), dynamic programming (DP) and non-linear programming NLP. Deterministic models use a specific sequence of streamflows to determine operating policies. Stochastic models, on the other hand, rely on statistical descriptions of the streamflow and forecast process to obtain operating policies. In this paper, a stochastic dynamic programming SDP formulation is adopted because it can handle stochastic inflows, non-linear objective functions and constraints, and a large number of stages. In addition, since the problem only involves one reservoir, the curse of dimensionality found in SDP is not an issue. See Labadie (2004) for a recent review of these techniques.

In SDP release decisions are made to maximize (minimize) current benefits (costs) plus the expected benefits (costs) from future operation, which are represented by the recursively calculated cost-to-go function $F^{*}$. In reservoir operation studies, the most common state variables are the volume of water in the reservoirs and a description of current hydrologic conditions (Tejada-Guibert et al., 1995). Let $n$ be the number of stages remaining until the end of the planning horizon, $r_{t}$ be the release during period $t, a_{t}$ the inflow during period $t, s_{t}$ the storage at the beginning of time period $t$, $E$ the expectation operator, $\alpha$ the discount factor, and $g_{t}$ the system operational benefit during period $t$.

Considering the reservoir operation problem as periodic and infinite-horizon the long-term optimal operating policy can be found by the recursive solution of the functional SDP equation

$$
\begin{aligned}
F_{n}^{*}\left(s_{t}, a_{t}\right) & =\max _{r_{t}}\left\{g_{t}\left(s_{t}, a_{t}, r_{t}\right)\right. \\
& \left.+\underset{a_{t+1} \mid a_{t}}{E} \alpha F_{n-1}^{*}\left(s_{t+1}, a_{t+1}\right)\right\}
\end{aligned}
$$


subject to

$s_{t+1}=s_{t}+a_{t}-r_{t}-l_{t}-e_{t}\left(s_{t}\right)-i_{t}$

$s_{\min } \leq s_{t+1} \leq s_{\max }$

$r_{\min } \leq r_{t} \leq r_{\max }$

where $s_{\min }$ and $s_{\max }$ are the lower and upper bounds on storage, $r_{\min }$ and $r_{\max }$ are the lower and upper bounds on release, $l_{t}$ are the spillage losses, $i_{t}$ are lateral irrigation withdrawals, $e_{t}$ are the evaporation losses.

The recursive Eq. (11) is carried out until the change in the cost-to-go function from one iteration to the next becomes nearly constant for each point of the discrete state space domain (Loucks and Beek, 2005). The resulting steady-state release policy $r_{t}^{*}$ and cost-to-go functions $F_{n}^{*}$ constitute the sets of solutions that can be used by reservoir operators to derive an optimal release policy. In this study, the time span of a stage is one month, and the period is one year. In practice, to solve the SDP model (Eq. 11), the hydrologic state and the storage variables must be discretized into $n_{a}$ and $n_{s}$ intervals respectively, each represented by a characteristic value (Yakowitz, 1982). The continuous domain $\left(a_{t}, s_{t}\right)$ is thus replaced by a grid so that an approximate solution of (Eq. 11) can be developed by evaluating (11) at the grid points only. Here, 12 grids of 110 points are used $\left(n_{a}=5\right.$ and $n_{s}=22$ ), which is consistent with the discretization schemes adopted in other studies (Kim and Palmer, 1997; TejadaGuibert et al., 1995; Karamouz and Vasiliadis, 1992).

When the algorithm moves from one stage (e.g. $n-1)$ to the next (e.g. $n$ ), the values of $F_{n-1}$ are available only at the grid points. However, in order not to restrict the release decisions $r_{t}$ to the trajectories imposed by the grid points, the cost-to-go functions $F_{n-1}^{*}$ are approximated by cubic splines, that is, individual multivariate cubic polynomials defined on each subregion of the state space domain delimited by the grid points. The spline coefficients are determined by requiring the spline to interpolate $F_{n-1}^{*}$ at each point of the state space domain (Johnson et al., 1993). The interpolations $F_{n-1}^{*}$ are only carried out with respect to the storage dimension since the hydrologic state is still described by transition probabilities.

In this study, the release decisions are determined by a variant of SDP, called FSDP, in which the objective function of the recursive equation is no longer an economic one, but rather corresponds to a FCSP in which the flexible constraints encode the satisfaction levels of the different water users and objectives. It must be stressed here that the physical constraints are still modeled by hard constraints. Denote $\mu_{O_{j, t}}\left(s_{t}, a_{t}, r_{t}\right)$ as the satisfaction of the $j$ th water user/objective at time $t$ when the storage is $s_{t}$, the inflow is $a_{t}$ and the decision is $r_{t}$. Let $\omega_{j, t}$ be the relative importance of the $j$ th water user/objective at time $t$ with $\sum_{j} \omega_{j, t}=1$. In addition, let $D_{n}\left(s_{t}, a_{t}\right)$ be the expected satisfaction-to-go from the optimal operation of the system from the current period $t$
( $n$ remaining stages) to the end of the planning horizon given that the system's state in period $t$ is $\left(s_{t}, a_{t}\right)$. The steady-state release policies are found by solution of:

$$
\begin{aligned}
D_{n}^{*}\left(s_{t}, a_{t}\right)= & {\left[\operatorname { m a x } _ { r _ { t } } \left\{\sum_{j=1}^{J-1} \omega_{j, t} \mu_{O_{j, t}}^{p}\left(s_{t}, a_{t}, r_{t}\right)+\right.\right.} \\
& \left.\left.\sum_{a_{t+1}} \rho\left(a_{t+1} \mid a_{t}\right) \omega_{J, t}^{*_{n-1}^{*}}\left(s_{t+1}, a_{t+1}\right)\right\}\right]^{p^{-1}}
\end{aligned}
$$

where $\rho($.$) are flow transition probabilities estimated from$ historical flow records.

Similar approaches can be found in Fontane et al. (1997); Esogbue and Kaprzyk (1998); Tilmant et al. (2002b) to derive operating policies of multipurpose reservoirs with imprecise operating objectives. A comparison between FSDP and SDP derived policies is described in Tilmant et al. (2002a).

In this study, the so-called Flexible SDP (FSDP) functional Eq. (15) will be used to derive reservoir operating policies corresponding to different group decision-making approaches as encountered in social choice theory. Several FSDP models will be developed with $p=1, p \rightarrow \infty$ and $p \rightarrow-\infty$ to determine optimal release decisions and then simulate the operation of the multipurpose reservoir with these three different rules. If the multiplicative variant of AHP is used, then the aggregation function would correspond to Eq. (8), i.e. the weighted geometric mean, and the FSDP) model woud be implemented with $p=0$. Meaningful performance indicators, such as the reliability in meeting irrigation water demands, will then be estimated from simulation results and used to compare the three FSDP formulations corresponding to three different conceptions of collective choice.

\section{Case study}

This reservoir is located in the Maule river basin near Santiago in the central part of Chile (Fig. 4). Water in the basin was used primarily for irrigation and more recently for hydropower generation. The Colbùn hydropower plant is dispatched together with the other power plants, both thermal and hydro, of the Sistema Interconectado Central, one of the Chilean electricity systems, by an Independant System Operator (ISO). The ISO produces a dispatch based on a least-cost criterion (also called "merit-order" operation): hydropower plants are dispatched so as to minimize the expected operating costs of the hydrothermal electrical system over a given planning period (here 4 years). Hence, despite the fact that this reservoir is not managed directly by its water users, it is used here for illustrative purposes i.e. to compare the performances generated by different operating policies corresponding to different group decision-making approaches. Hence, no conclusion can be drawn regarding the historical performances. The main characteristics of the Colbùn reservoir are listed in Table 1. 


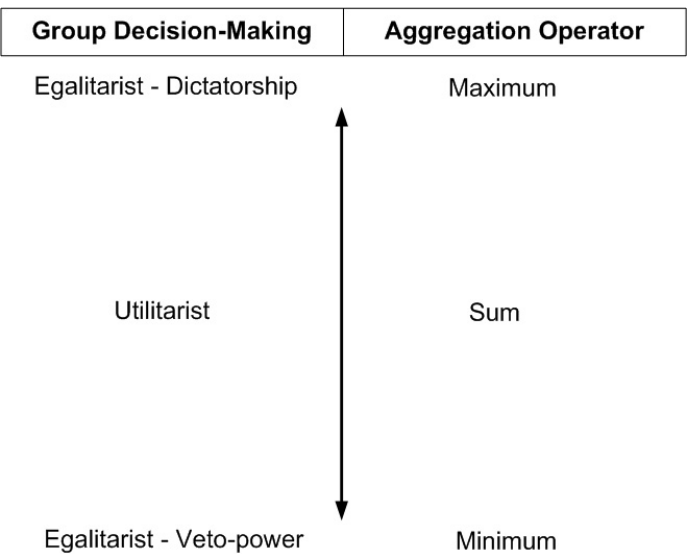

Fig. 1. Group decision-making approaches and their aggregation operators

Table 1. Main characteristics of Colbùn reservoir.

\begin{tabular}{ll}
\hline Max storage capacity $\left[\mathrm{hm}^{3}\right]$ & 1515 \\
Dead storage capacity $\left[\mathrm{hm}^{3}\right]$ & 380 \\
Installed capacity [MW] & 400 \\
Expected annual production [GWh] & 2500 \\
Max turbined release $\left[\mathrm{m}^{3} / \mathrm{s}\right]$ & 310 \\
Min head [m] & 129 \\
Max head [m] & 168 \\
Average annual inflow $\left[\mathrm{hm}^{3} / \mathrm{yr}\right]$ & 7492 \\
Max annual inflow $\left[\mathrm{hm}^{3} / \mathrm{yr}\right]$ & 12192 \\
Min annual inflow $\left[\mathrm{hm}^{3} / \mathrm{yr}\right]$ & 2541 \\
Irrigation water demand $[\mathrm{hm} / \mathrm{yr}]$ & 3457 \\
\hline
\end{tabular}

In the Maule river basin the development of hydropower has been a source of tension between irrigators and the hydropower companies because:

- the operation of the hydropower plants affects the hydrological regime therefore violating the prior rights of farmers for constant flows;

- water diverted for hydropower generation does not immediately return to the river therefore bypassing many farmers.

To mitigate the impact on farmers, hydropower companies have constructed additional hydraulic infrastructures (irrigation offtakes, canals). This has had practical implications for the management of the Colbùn reservoir as the elevation of a major irrigation canal imposes a lower limit on the storage level therefore reducing the usable storage capacity by as much as $30 \%$ (from $1515 \mathrm{hm}^{3}$ to $950 \mathrm{hm}^{3}$ ). In addition, hydropower companies are also bound to respect pre-defined monthly release targets reflecting agricultural demands. Consequently, the number of objectives $J$ for the Colbùn reservoir is four: (1) hydropower generation $\left(O_{1}\right)$,
Table 2. Reservoir objectives.

\begin{tabular}{|c|c|c|c|c|}
\hline & $\begin{array}{l}\text { Hydropower } \\
\text { generation } \\
\mu_{O_{1, t}}\end{array}$ & $\begin{array}{l}\text { Storage } \\
\text { Control } \\
\mu_{O_{2, t}}\end{array}$ & $\begin{array}{l}\text { Downstream } \\
\text { irrigation } \\
\mu_{O_{3, t}}\end{array}$ & $\begin{array}{l}\text { Future } \\
\text { operation } \\
D_{n-1}\end{array}$ \\
\hline$r_{t}$ & + & + & + & + \\
\hline$s_{t}$ & + & + & & \\
\hline$r_{t+1}$ & & & & + \\
\hline$s_{t+1}$ & + & & & + \\
\hline
\end{tabular}

(2) storage control for flood prevention during the high flow season and lateral irrigation during the low flow season $\left(\mathrm{O}_{2}\right)$, (3) downstream irrigation $\left(\mathrm{O}_{3}\right)$, and (4) the need to ensure satisfactory continuing operation, i.e. to avoid future shortfalls in any of the first three operating objectives $\left(\mathrm{O}_{4}\right)$. Table 2 lists the objectives and their associated control/state variables.

The general FSDP model for the Colbùn reservoir can be written as:

$$
\begin{aligned}
D_{n}^{*}\left(s_{t}, a_{t}\right)= & {\left[\operatorname { m a x } _ { r _ { t } } \left\{\sum_{j=1}^{3} \omega_{j, t} \mu_{O_{j, t}}^{p}\left(s_{t}, a_{t}, r_{t}\right)+\right.\right.} \\
& \left.\left.\sum_{a_{t+1}} \rho\left(a_{t+1} \mid a_{t}\right) \omega_{4, t} D_{n-1}^{*^{p}}\left(s_{t+1}, a_{t+1}\right)\right\}\right]^{p^{-1}}
\end{aligned}
$$

with the same restrictions on the state and decision variables as in Eq. (11). The membership functions $\mu_{O_{j}}$ and weighting coefficients $\omega_{i}$ were derived from existing operating rules as well as from discussions with experts familiar with the system. A first set of weights was generated with AHP using the following information:

- $a_{2,3}=a_{3,2}=1$ means downstream irrigation $O_{3}$ and storage control $\mathrm{O}_{2}$ are equally important for equity reasons (because both serve irrigators)

- $a_{2,1}=a_{3,1}=3$ means $O_{3}$ and $O_{2}$ are moderately more important than hydropower $O_{1}$

- $a_{2,4}=a_{3,4}=5$ means $O_{3}$ and $O_{2}$ are strongly more important than $\mathrm{O}_{4}$

- $a_{1,4}=3$ means $O_{1}$ is moderately more important than $\mathrm{O}_{4}$

The consistency ratio of the corresponding intensity matrix $A$ is 0.016 , which is considered acceptable as it is lower than 0.1 , and the "theoretical" weight vector is [0.15 0.390 .390 .07$]$. This weight vector was then adjusted so as to better reflect the subjective assessment of the relative importances by the three experts familiar with the system: the sum of the weights of $O_{2}$ and $O_{3}$ should not exceed 0.7 and the difference was spread over $O_{1}$ and $O_{4}$. The adjusted weight vector is [ 0.180 .350 .350 .12$]$.

The shape of the membership function is also an important factor that will affect the results. Here, the membership function for the hydropower objective is the hydroelectric production function normalized on the unit interval as depicted on 


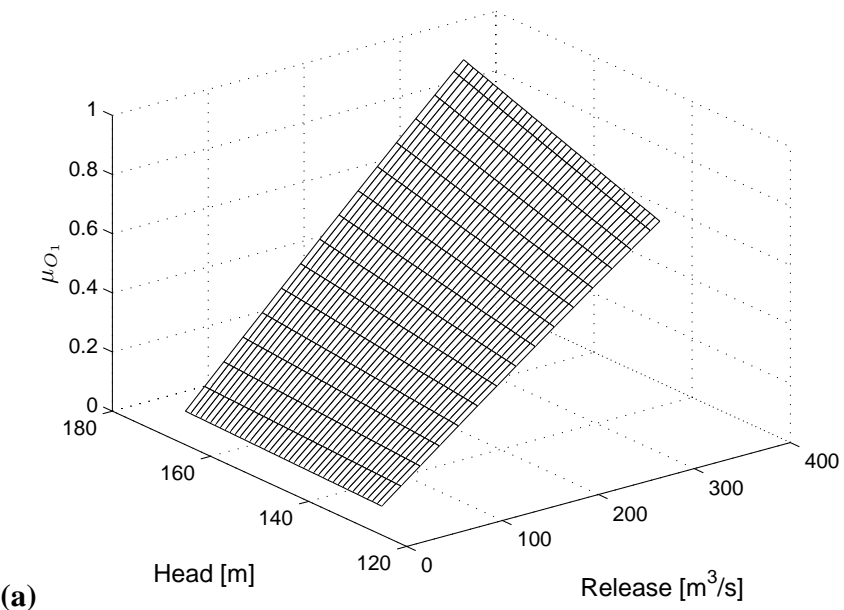

(a)

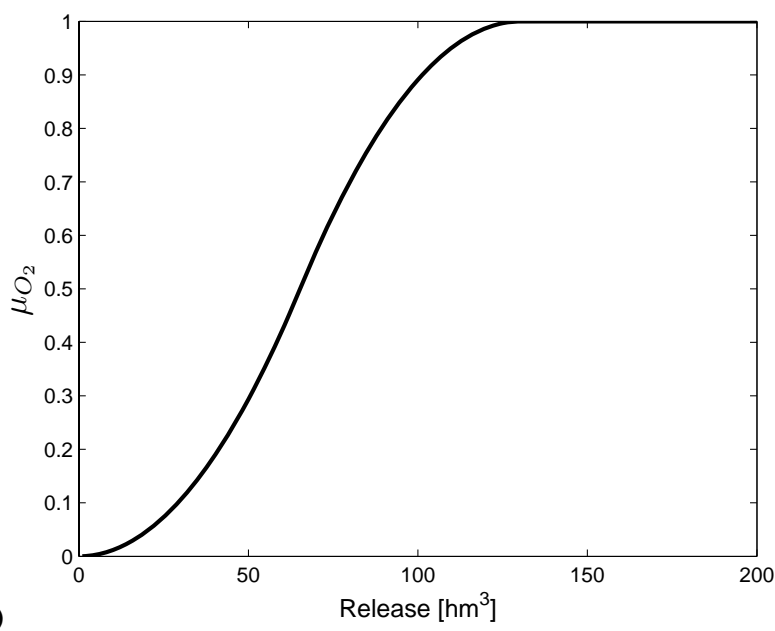

(b)

Fig. 2. Examples of membership functions: (a) hydropower objective, (b) downstream irrigation objective in March.

Table 3. Aggregation parameters.

\begin{tabular}{llll}
\hline & $\begin{array}{l}\text { Egalitarian } \\
\text { Veto-power } \\
\text { Model I }\end{array}$ & Utilitarian & $\begin{array}{l}\text { Egalitarian } \\
\text { Dictatorship } \\
\text { Model III }\end{array}$ \\
\hline$p$ & $p \rightarrow-\infty$ & $p=1$ & $p \rightarrow+\infty$ \\
$\omega_{j}$ & no & yes & no \\
\hline
\end{tabular}

Fig. 2. An example of a membership function for the irrigation objective is given in Fig. 2.

As mentioned above, this formulation is implemented with three different aggregation operators corresponding to utilitarian or egalitarian approaches to collective choice. There are therefore three FSDP models as listed in Table 3.

The FSDP-MODEL I corresponds to the egalitarian approach to group decision-making in which each water user has a veto power. As pointed out early, this approach is also the most equitable as the decision maximizes the satisfaction of the least-satisfied water user.
Table 4. Simulated performances.

\begin{tabular}{llll}
\hline & $\begin{array}{l}\text { Egalitarian } \\
\text { Veto-power } \\
\text { Model I }\end{array}$ & Utilitarian & $\begin{array}{l}\text { Egalitarian } \\
\text { Dictatorship } \\
\text { Model III }\end{array}$ \\
\hline$\delta[\%]$ & 68 & 70 & 74 \\
$\beta[\%]$ & 95 & 85 & 34 \\
$\gamma[\mathrm{GWh}]$ & 2195 & 2214 & 2247 \\
\hline \multicolumn{4}{c}{} \\
$D_{n}^{*}\left(s_{t}, a_{t}\right)=\max _{r_{t}} \min _{\mu, D}\left\{\mu_{O_{j, t}}\left(s_{t}, a_{t}, r_{t}\right), \ldots\right.$, \\
$\left.\sum_{a_{t+1}} \rho\left(a_{t+1} \mid a_{t}\right) D_{n-1}^{*}\left(s_{t+1}, a_{t+1}\right)\right\}$
\end{tabular}

The FSDP-MODEL II corresponds to the utilitarian approach to group decision-making in which each water user no longer has a veto power but where their relative importance is now considered. Here, the optimal solutions $r^{*}\left(s_{t}, a_{t}\right)$ are all Pareto optimal and compensation between high and low satisfaction degrees is now permitted, which implies that most water users will perceive these solutions as a "zero-sum" game.

$$
\begin{aligned}
D_{n}^{*}\left(s_{t}, a_{t}\right)= & \\
& \max _{r_{t}}\left\{\sum_{j=1}^{3} \omega_{j, t} \mu_{O_{j, t}}\left(s_{t}, a_{t}, r_{t}\right)+\right. \\
& \left.\sum_{a_{t+1}} \rho\left(a_{t+1} \mid a_{t}\right) \omega_{4, t} D_{n-1}^{*}\left(s_{t+1}, a_{t+1}\right)\right\}
\end{aligned}
$$

Finally, the FSDP-MODEL III corresponds to the egalitarian approach to group decision-making in which each water user is a potential dictator. Although not desirable, this approach is considered here for comparison purposes.

$$
\begin{aligned}
D_{n}^{*}\left(s_{t}, a_{t}\right)= & \\
& \max _{r_{t}} \max _{\mu, D}\left\{\mu_{O_{j, t}}\left(s_{t}, a_{t}, r_{t}\right), \ldots,\right. \\
& \left.\sum_{a_{t+1}} \rho\left(a_{t+1} \mid a_{t}\right) D_{n-1}^{*}\left(s_{t+1}, a_{t+1}\right)\right\}
\end{aligned}
$$

FSDP-derived operating rules are then used in simulation. Simulation results consist of the end-of-the month storage level, the monthly release through the turbines, the spillage and evaporation losses, and the average monthly hydroelectric production. Each simulation run starts with initial volume in storage of $1449 \mathrm{hm}^{3}$, i.e. a reservoir almost full, and uses historical monthly inflows recorded during the 19882000 period.

\section{Simulation results}

The performance indicators that are used to investigate the impact of the different group decision-making approaches on the performance of the Colbùn reservoir are

- the reliability in meeting downstream irrigation demands $\delta=P\left(s_{t} \geq s_{T}\right)$ where $s_{T}$ is the minimum allowable storage volume for lateral irrigation 


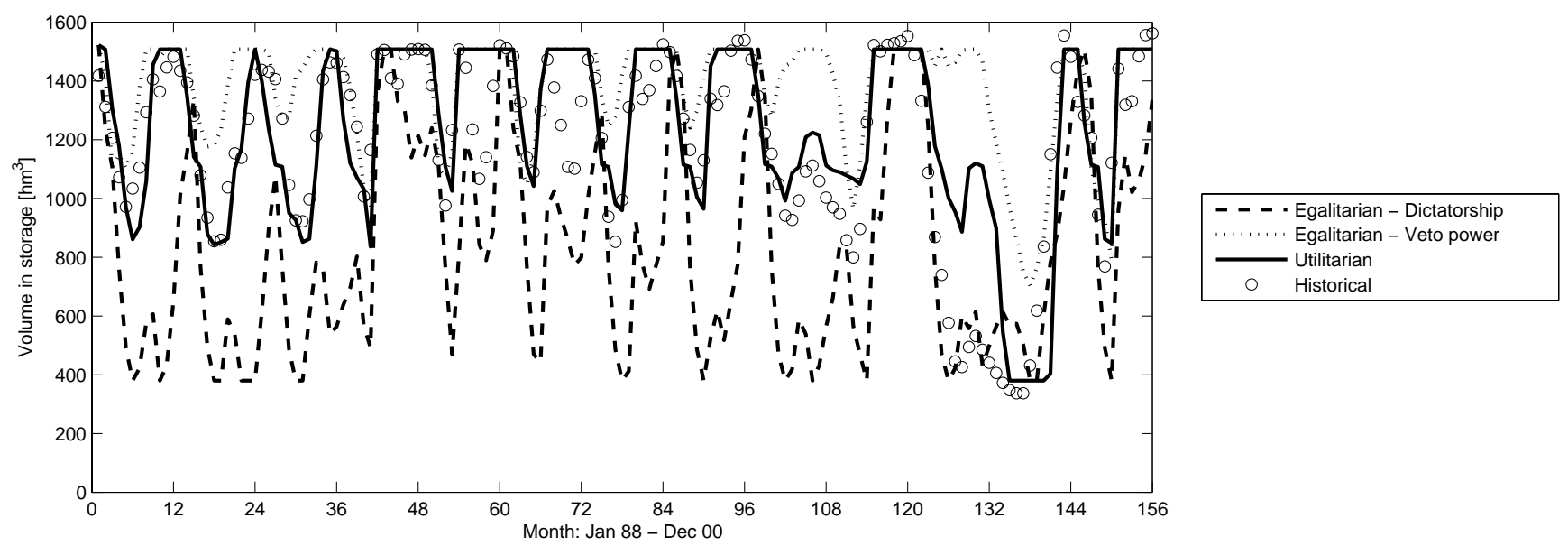

Fig. 3. Simulated storage levels for different group-decision making approaches.

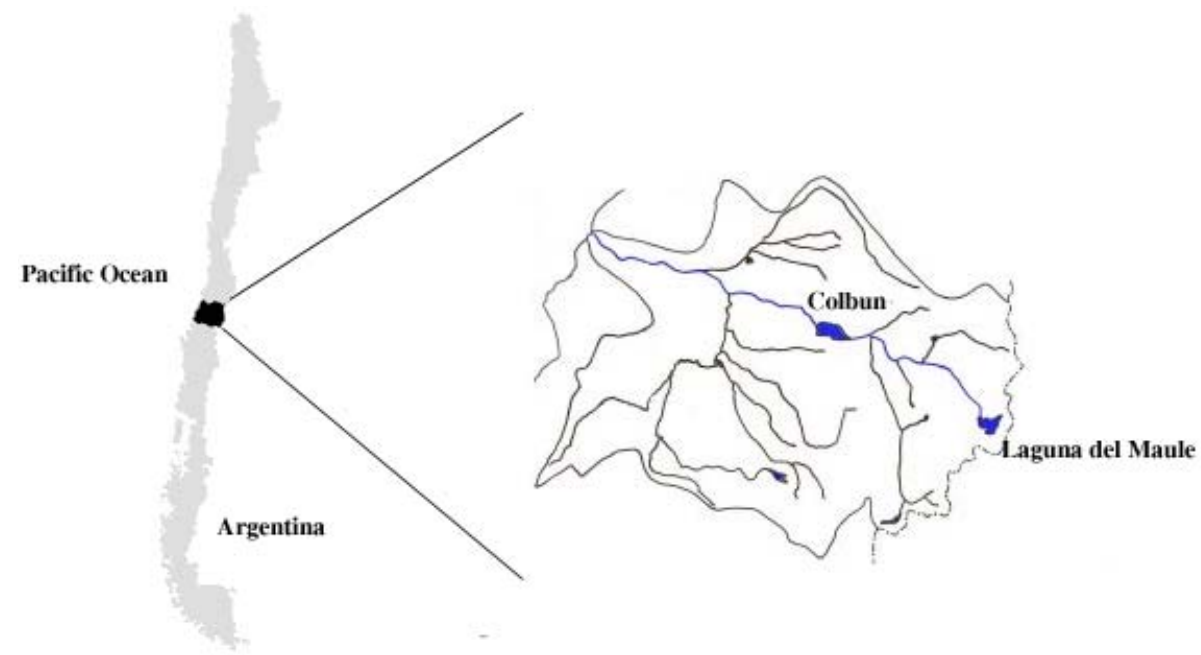

Fig. 4. The Maule river basin in Chile.

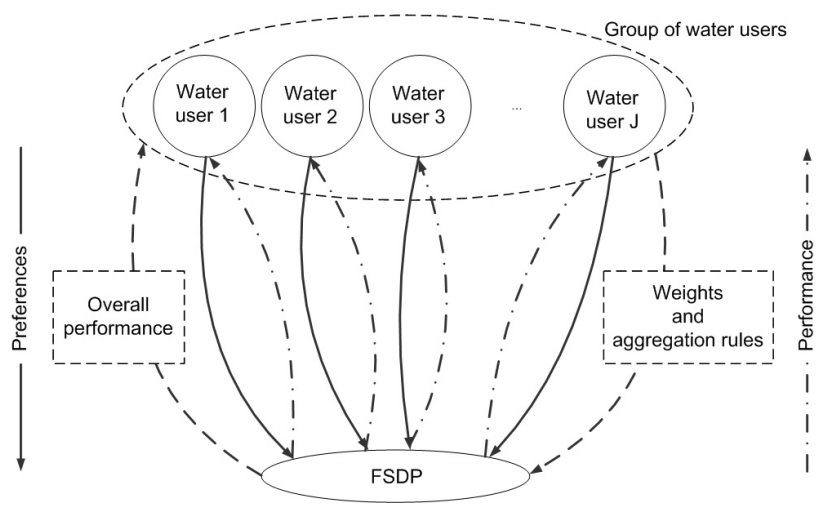

Fig. 5. Representation of the FSDP methodology.
- the reliability in delivering water for lateral irrigation $\beta=P\left(r_{t} \geq d_{t}\right)$ where $d_{t}$ is the target release for downstream irrigation in period $t$

- (iii) the average annual production of hydroelectricity $\gamma$.

Simulated performances corresponding to the three FSDP models and to the "88-00" time period are listed in Table 4 .

The examination of Table 4 and Fig. 3 reveals that the three conceptions of collective choice yield different release policies. The egalitarian approach with dictatorship tend to favor high releases therefore lowering the volume in storage. The reason is to be found in that this conception of collective choice concentrates on the satisfaction of the most satisfied decision maker, which is here either the hydropower 
company or downstream farmers whose satisfaction degrees $\mu_{O_{1}}$ are in general larger than that of the two other objectives $\left(\mu_{O_{1}}, \mu_{O_{3}}>\mu_{O_{2}}, \mu_{O_{4}}\right)$ because hydropower generation increases more rapidly with the release term rather that with the head term. The opposite behavior is observed with the egalitarian approach with veto power. As a matter of fact, this approach encourages high pool elevation throughout the year as both the lateral irrigation and future operation objectives are likely to be the least satisfied objectives if aggressive release are implemented to meet downstream irrigation water demands and the production of hydroelectricity.

In between these two extremes, the utilitarian approach generates more balanced release policies, which is consistent with the fact that trade-offs are now considered between aggressive and conservative objectives. This approach explicitly recognizes that high satisfaction levels can compensate lower satisfaction levels in order to derive a policy that compromises between the two extreme egalitarian approaches to collective choice. The simulation results speak for themselves: (i) the storage levels corresponding to the utilitarian approach lie often somewhere in between the storage levels corresponding to the two egalitarian approaches, and (ii) this is confirmed by the performance indicators as $\left(\delta_{I}<\delta_{I I}<\delta_{I I I}\right)$ and $\left(\beta_{I}>\beta_{I I}>\beta_{I I I}\right)$. The same observation can be made for the average annual production of hydroelectricity (with $\gamma_{I}>\gamma_{I I}>\gamma_{I I I}$ ). The third model generates more energy because it better exploits the volume in storage with a drawdown/refill cycle that takes place over the entire reservoir.

In practice, the proposed methodology can be seen as a loop in which, on one hand, waters users provide their preferences and, on the other hand, these preferences are processed by the FSDP model which then provides water users with short and long-term consequences. These consequences must be easily interpreted by water users thanks to the use of several indicators such as satisfaction, reliability, resiliency, economic performance (costs vs. benefits, valueat-risk, cashflow-at-risk), etc. The interpretation should then encourage discussion between water users and ultimately generates a new set of preferences, and thus a new loop in Fig. 5, which would lead to consensual solutions.

\section{Conclusions}

This paper illustrates the impact of various conceptions of collective choice on the performance of a water resource system. The group decision-making process is mathematically formulated as a flexible constraint satisfaction problem which assumes both quantification and commensurability of preferences. From these demanding assumptions, an aggregation operator must be carefully selected to identify consensual solutions. Results show that extreme conceptions of collective choice, i.e. veto-power and dictatorship, generate extreme release policies. Utilitarian approaches appear to be more consistent even though compensatory effects between low and high satisfaction levels are not easily accepted by water users.

The proposed model can provide some insights into how the decision-making process will affect current and future allocations and ultimately the satisfaction of the users. Various risk indicators can also be derived from long-term simulations which capture the inherent uncertainties attached to this water resource management problem. The model can also be used to arbitrate between efficiency and equity principles for allocating water among competing users.

Potential refinements include (i) the improvement of aggregation operators, (ii) the consideration of "public" water services, i.e. the operating objectives that can be viewed as public goods (e.g. flood control) and their aggregation with "individual" objectives (e.g. hydropower, navigation), and (iii) the elicitation of preferences. Further researches will concentrate on other decentralized allocation mechanisms such as capacity sharing (Tilmant and van der Zaag, 2006).

Edited by: D. Savic

\section{References}

Bellman, R. and Zadeh, L.: Decision-making in a fuzzy environment, Management Sciences, 17, 141-164, 1970.

Beroggi, G.: Decision modeling in policy management - An introduction to the analytic concepts, Kluwer Academic Publisher, Dordrecht, The Netherlands, 1999.

Choo, E., Schoner, B., and Wedley, W.: Interpretation of criteria weights in multicriteria decision making, Computer and Industrial Engineering, 37, 527-541, 1999.

Dinar, A., Rosegrant, M., and Meinzen-Dick, R.: Water allocation mechanisms: Principles and examples, World Bank Technical Paper, 1997.

Dubois, D. and Fortemps, P.: Computing improved optimal solutions to max-min flexible constraint satisfaction problems, European J. Operational Res., 118, 95-126, 1999.

Dubois, D., Fargier, H., and Prade, H.: Refinements of the maximin approach to decision-making in a fuzzy environement, Fuzzy sets and systems, 81, 103-122, 1996.

Esogbue, A. and Kaprzyk, J.: Fuzzy dynamic programming, in: Handbook of fuzzy sets series, vol. 5, pp. 281-307, Kluwer Academic Publishers, Boston, 1998.

Fontane, D., Gates, T., and Moncada, E.: Planning reservoir operation with imprecise objectives, J. Water Resour. Planning Manage., 123, 154-163, 1997.

Forman, E. and Peniwati, K.: Aggregating individual judgments and priorities with the Analytic Hierarchy Process, European J. Operational Res., 108, 165-169, 1998.

Hjorth, P., Kundzewicz, W., Kutchment, L., and Rosbjerg, D.: Critiques of present reservoirs, in: Sustainable Reservoir Development and Management, edited by: IAHS Publication, nbr 251, pp. 125-247, Wallingford, UK, 1998.

Honert, R. V. D. and Lootsma, F.: Group preference aggregation in the multiplicative AHP - The model of the group decision 
process and Pareto optimality, European J. Operational Res., 96, 363-370, 1996.

Johnson, S., Stedinger, J., Shoemaker, J., Li, C., and TejadaGuibert, A.: Numercial solution of continuous-state dynamic programs using linear and spline interpolation, Operation Res., 41, 484-500, 1993.

Karamouz, M. and Vasiliadis, H.: Bayesian Stochastic Optimization of Reservoir Operation Using Uncertain Forecasts, Water Resour. Res., 28, 1221-1232, 1992.

Kaymak, U. and van Nauta Lemke, H.: A sensitivity analysis approach to introducing weight factors into decision functions in fuzzy multicriteria decision making, Fuzzy sets and systems, 97, 169-182, 1998.

Kim, Y. and Palmer, R.: Value of Seasonal Flow Forecasts in Bayesian Stochastic Programming, J. Water Resour. Plann. Manage., 123, 327-335, 1997.

Labadie, J. W.: Optimal operation of multireservoir systems: Stateof-the-Art review, J. Water Resour. Plann. Manage., 130, 93-111, 2004.

Lootsma, F.: A model for the relative importance of the criteria in the Multiplicative AHP and SMART, European J. Operational Res., 94, 467-476, 1996.

Loucks, D. and Beek, E. V.: Water resources systems planning and management, UNESCO Publishing, Paris, France, 2005.

Ramanathan, R.: A note on the use of the analytic hierarchy process for environmental impact assessment, European J. Operational Res., 63, 27-35, 2001.

Rawls, J.: A theory of Justice, Cambridge University Press, Mass, USA, 1970
Saaty, T.: The analytic hierarchy process, McGraw-Hill, New York, 1980.

Sagoff, M.: Aggregation and deliberation in valuing environmental public goods: A look beyond contingent pricing, Ecological Economics, 24, 213-230, 1998.

Slany, W.: Scheduling as a fuzzy multiple criteria optimization problem, Fuzzy sets and systems, 78, 197-222, 1996.

Srdjevic, B.: Linking analytic hierachy process and social choice methods to support group decision-making in water management, Decision Support System, 42(4), 2261-2273, 2007.

Tejada-Guibert, A., Johnson, S., and Stedinger, J.: The Value of Hydrologic Information in Stochastic Dynamic Programming Models of a Multireservoir System, Water Resour. Res., 31, 25712579, 1995.

Tilmant, A. and van der Zaag, P.: Towards good governance of hydraulic works: A review of management instruments, in: EGU Meeting, Vienna, Austria, 2006.

Tilmant, A., Duckstein, L., Persoons, E., and Vanclooster, M.: Comparison of fuzzy and non-fuzzy optimal reservoir operating policies, J. Water Resour. Plann. Manage., 128, 390-398, 2002a.

Tilmant, A., Fortemps, P., and Vanclooster, M.: Effect of averaging operators in fuzzy optimisation of reservoir operation, Water Resour. Manage., 16, 1-22, 2002b.

WCD: World Commission on Dams. Dams and Development: A New Framework for Decision-making, Earthscan Publications Ltd, London and Sterling, VA, 2000.

Yakowitz, S.: Dynamic programming application in water resources, Water Resour. Res., 18, 673-696, 1982.

Zimmermann, H.: Fuzzy set theory and its applications, Kluwer, Dordrecht, 1991. 